\title{
Case Report \\ Sporotrichoid-Like Spread of Cutaneous Mycobacterium chelonae in an Immunocompromised Patient
}

\author{
Daria Marley Kemp, ${ }^{1}$ Anusha G. Govind, ${ }^{2}$ Jun Kang, ${ }^{1}$ \\ Caroline C. Brugger, ${ }^{2}$ and Young C. Kauh ${ }^{1}$ \\ ${ }^{1}$ Department of Dermatology and Cutaneous Biology, Thomas Jefferson University, Philadelphia, PA, USA \\ ${ }^{2}$ Department of Infectious Disease, Thomas Jefferson University, Philadelphia, PA, USA \\ Correspondence should be addressed to Anusha G. Govind; anusha.govind@jefferson.edu
}

Received 5 June 2017; Accepted 20 July 2017; Published 23 August 2017

Academic Editor: Kowichi Jimbow

Copyright (C) 2017 Daria Marley Kemp et al. This is an open access article distributed under the Creative Commons Attribution License, which permits unrestricted use, distribution, and reproduction in any medium, provided the original work is properly cited.

\begin{abstract}
Mycobacterium chelonae is a rapidly growing mycobacterium found in water and soil that can cause local cutaneous infections in immunocompetent hosts but more frequently affects immunocompromised patients. Typically, patients will present with painful subcutaneous nodules of the joints or soft tissues from traumatic inoculation. However, exhibiting a sporotrichoid-like pattern of these nodules is uncommon. Herein, we report a case of sporotrichoid-like distribution of cutaneous Mycobacterium chelonae in a patient with systemic lupus erythematosus on significant immunosuppressive medications. Clinicians treating immunocompromised patients should be cognizant of their propensity to develop unusual infections and atypical presentations.
\end{abstract}

\section{Case Presentation}

A 54-year-old female, with a history of systemic lupus erythematosus on mycophenolate mofetil (3 g daily), prednisone (10 $\mathrm{mg}$ daily), and cyclosporine (50 $\mathrm{mg}$ twice a day), presented with a 4-month duration of unilateral painful wellcircumscribed erythematous to violaceous subcutaneous nodules extending from her 2nd finger web space to her dorsal wrist and forearm in a sporotrichoid-like pattern (Figure 1(a)). She denied exposure to fish tanks, swimming pools, tattoo needles, gardening, fresh or brackish waters, or nail salons.

Dorsal wrist nodule biopsy revealed suppurative granulomatous inflammation (Figures 2(a) and 2(b)). Both acidfast bacilli (AFB) and Fite's acid-fast stains (Figure 2(c)) showed bacilli in the dermis, consistent with a mycobacterial infection. AFB culture and stain were positive for the rapid grower, Mycobacterium chelonae. Initially, she was started on dual antibiotic regimen of linezolid $600 \mathrm{mg}$ twice daily and azithromycin $250 \mathrm{mg}$ daily. Subsequently, she was converted to linezolid $600 \mathrm{mg}$ and clarithromycin $250 \mathrm{mg}$ twice daily once susceptibilities returned and also due to gastrointestinal upset from azithromycin. Although the patient had no adverse reaction to linezolid, the dose was decreased to $600 \mathrm{mg}$ daily to ensure tolerability and continued normal blood counts. Given her active infection, rheumatology decreased her mycophenolate mofetil dose and discontinued cyclosporine. Improvement of her skin lesions was evident on follow-up within three months (Figure 1(b)). At four months, her nodules had fully resolved and therapy was discontinued.

\section{Discussion}

Mycobacterium chelonae is a rapidly growing mycobacterium commonly found in water and soil. The name chelonae is derived from the Greek word for turtle, chelōnē, as it was initially isolated in 1903 from sea turtles [1]. It can cause local cutaneous infections in immunocompetent patients, but infections are more frequently identified in patients taking immunosuppressive medications. Patients can develop painful subcutaneous nodules involving joints or soft tissue [2]. These skin nodules, as well as deeper and more disseminated infections, are usually from traumatic introduction. However, preceding trauma may not always be 


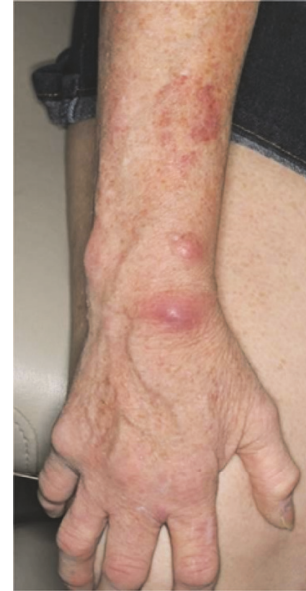

(a) Initial encounter

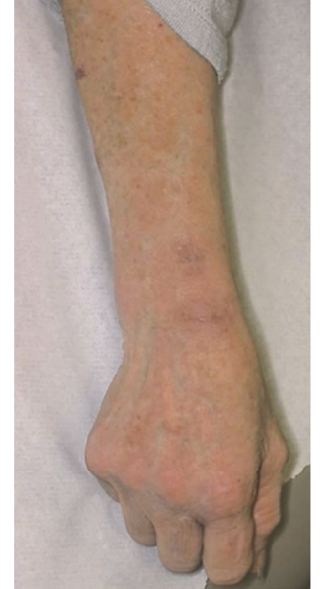

(b) 4-month follow-up

Figure 1: Clinical photos.

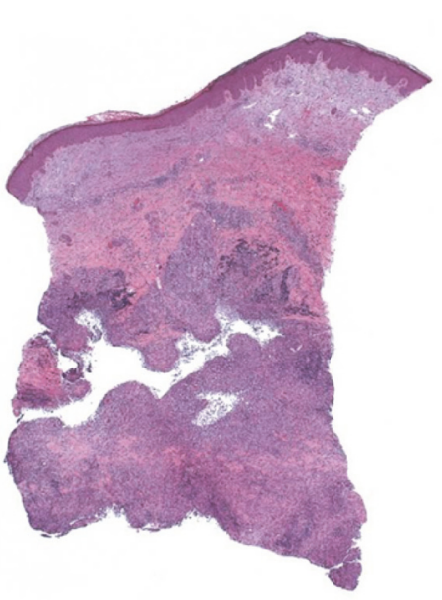

(a) Hematoxylin-eosin (40x)

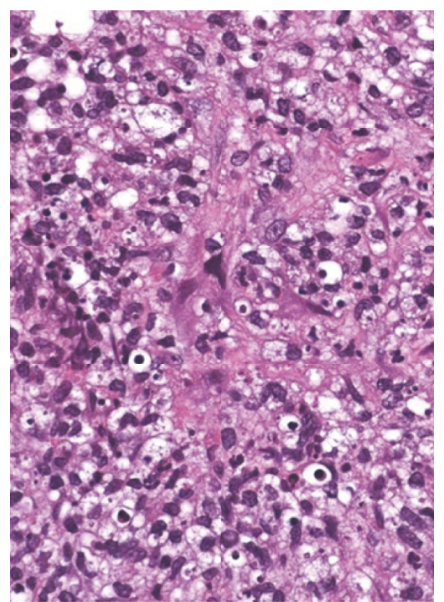

(b) Hematoxylin-eosin (400x)

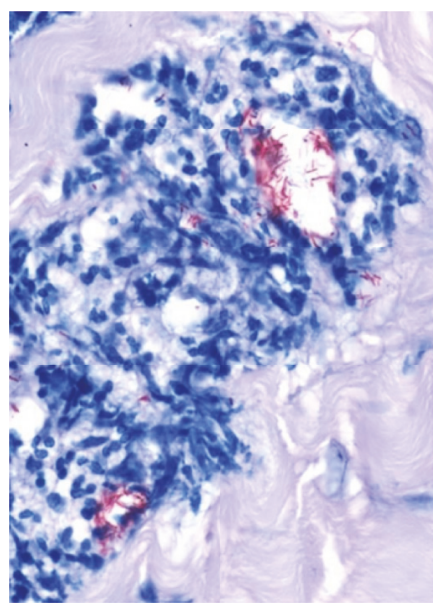

(c) Fite's acid-fast stain (400x)

Figure 2: Pathologic findings from a right wrist skin nodule: suppurative granulomatous dermatitis in deep dermis (a \& b) and numerous acid-fast bacilli highlighted by Fite's acid-fast stain (c).

readily apparent. Sporotrichoid spread is a linear ascending extension along lymphatic chains, generally found with deep fungal infections but can also be present in other organisms. [3]. In 1992, Wallace et al. presented a series of 53 cases of $M$. chelonae disseminated cutaneous infections ( $>5$ lesions), which were either well-circumscribed lesions or a "confluent mass of cellulitis with multiple draining fistulas." They were generally at the distal end of an extremity but did not form a linear or sporotrichoid pattern [4]. Differential diagnosis for skin nodules in a sporotrichoid-spread pattern includes Sporothrix schenckii, M. marinum, other nontuberculous mycobacteria, Nocardia, Leishmania, and Tularemia [5].

Clinical presentation of Mycobacterium chelonae is often influenced by the immunological status of the patient. Approximately 10 cases have been described in the literature with a sporotrichoid-like pattern of $M$. chelonae involving an immunosuppressed patient $[1,3,5-10]$ and at least two cases in a presumably immunocompetent patient [11]. Mycobacteria are referred to as AFB for the complex that forms during the histological staining process between the mycolic acid in the mycobacterial cell wall and dye, which is resistant to the decolorizing mineral acid. Compared to classic AFB staining, Fite's acid-fast stain uses a milder destaining acid, as well as less alcohol during the staining process, which it suitable for more delicate mycobacterium, such as leprosy bacillus [12].

Although clarithromycin was initially the drug of choice in treatment, increasing drug resistance makes it an unsuitable single agent. Treatment generally involves two antibacterial agents, particularly in immunocompromised patients. Linezolid has been shown to be an effective second medication; however, its adverse effects of bone marrow suppression warrant close monitoring [2]. Once daily dosing of linezolid $600 \mathrm{mg}$ has demonstrated a potential benefit of decreasing the risk of toxicity over a prolonged course of treatment. 
However, a recent retrospective study found that the efficacy and safety profile of linezolid $600 \mathrm{mg}$ once daily and $300 \mathrm{mg}$ once daily in the treatment of multidrug and extensively drug-resistant tuberculosis showed potentially fewer neurotoxicities than a lower dose of linezolid [13].

Given the large number of immunocompromised patients clinicians serve, one must be mindful of their increased susceptibility to unusual infections and atypical presentations. It is vital that patients with suspicious sporotrichoid cutaneous lesions should have a biopsy with routine bacterial culture, mycobacterial culture, fungal culture, prolonged culture hold, molecular testing for Nocardia, and pathology. Only a few literature cases reported of $M$. chelonae present with sporotrichoid-like spread, typically in immunocompromised patients.

\section{Abbreviations and Acronyms}
AFB: $\quad$ Acid-fast bacilli
M. chelonae: Mycobacterium chelonae
M. marinum: Mycobacterium marinum.

\section{Disclosure}

This case has been presented at the 2017 AAD Annual Meeting Gross \& Microscopic Symposium on Saturday, March 4, 2017.

\section{Conflicts of Interest}

The authors declare that there are no conflicts of interest regarding the publication of this paper.

\section{Acknowledgments}

Jason B. Lee, M.D., Department of Dermatology and Cutaneous Biology, Thomas Jefferson University Hospital, provided the histopathological photos.

\section{References}

[1] E. Orrin, F. Worsnop, and J. Natkunarajah, "Sporotrichoid Mycobacterium chelonae," Australasian Journal of Dermatology, vol. 57, no. 3, pp. 244-245, 2016

[2] P. Parize, A. Hamelin, N. Veziris et al., "Induction therapy with linezolid/clarithromycin combination for Mycobacterium chelonae skin infections in immunocompromised hosts," Journal of the European Academy of Dermatology and Venereology, vol. 30, no. 1, pp. 101-105, 2016.

[3] E. M. Higgins and C. M. Lawrence, "Sporotrichoid spread of Mycobacterium chelonei," Clinical and Experimental Dermatology, vol. 13, pp. 234-236, 1988.

[4] R. J. Wallace, B. A. Brown, and G. O. Onyi, "Skin, soft tissue, and bone infections due to Mycobacterium chelonae chelonae: importance of prior corticosteroid therapy, frequency of disseminated infections, and resistance to oral antimicrobials other than clarithromycin," Journal of Infectious Diseases, vol. 166, no. 2, pp. 405-412, 1992.

[5] M. N. Schwendiman, R. P. Johnson, and J. S. Henning, "Subcutaneous nodules with sporotrichoid spread," Dermatology Online JournaL, vol. 15, no. 5, p. 11, 2009.
[6] T. Demitsu, H. Nagato, T. Inoue et al., "Cutaneous Mycobacterium chelonae infection with bilateral sporotrichoid involvement," International Journal of Dermatology, vol. 40, no. 9, pp. 597-599, 2001.

[7] W. Godard, N. Cordier, A. Kazmierczak, and D. Lambert, "Sporotrichosis-like skin infection caused by Mycobacterium chelonei and cured by minocycline," Presse Medicale, vol. 15, no. 3, p. 120, 1986.

[8] K. E. Greer, G. P. Gross, and S. H. Martensen, "Sporotrichoid Cutaneous Infection due to Mycobacterium chelonei," Archives of Dermatology, vol. 115, no. 6, pp. 738-739, 1979.

[9] A. G. Jopp-McKay and P. Randell, "Sporotrichoid cutaneous infection due to Mycobacterium chelonei in a renal transplant patient," Australasian Journal of Dermatology, vol. 31, no. 2, pp. 105-109, 1990.

[10] M. E. Murdoch and I. M. Leigh, "Sporotrichoid spread of cutaneous Mycobacterium chelonei infection," Clinical and Experimental Dermatology, vol. 14, pp. 309-312, 1989.

[11] E. Rosón, I. García-Doval, C. De la Torre, A. Losada, C. Feal, and M. Cruces, "Sporotrichoid spread of Mycobacterium chelonae in a presumably immunocompetent patient," Acta DermatoVenereologica, vol. 82, no. 2, pp. 142-143, 2002.

[12] N. Wengenack, Mycobacteria part 1: Stains and culture. Mayo Medical Laboratories, http://www.mayomedicallaboratories.com/ articles/hot-topic/2014/10-15-mycobacteria-pt-1/index.html.

[13] W.-J. Koh, Y. R. Kang, K. Jeon et al., "Daily $300 \mathrm{mg}$ dose of linezolid for multidrug-resistant and extensively drug-resistant tuberculosis: Updated analysis of 51 patients," Journal of Antimicrobial Chemotherapy, vol. 67, no. 6, Article ID dks078, pp. 15031507, 2012. 


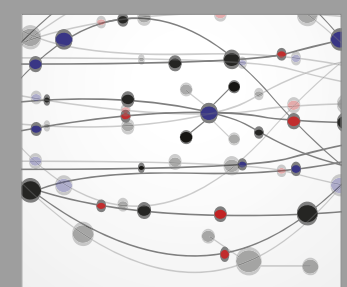

The Scientific World Journal
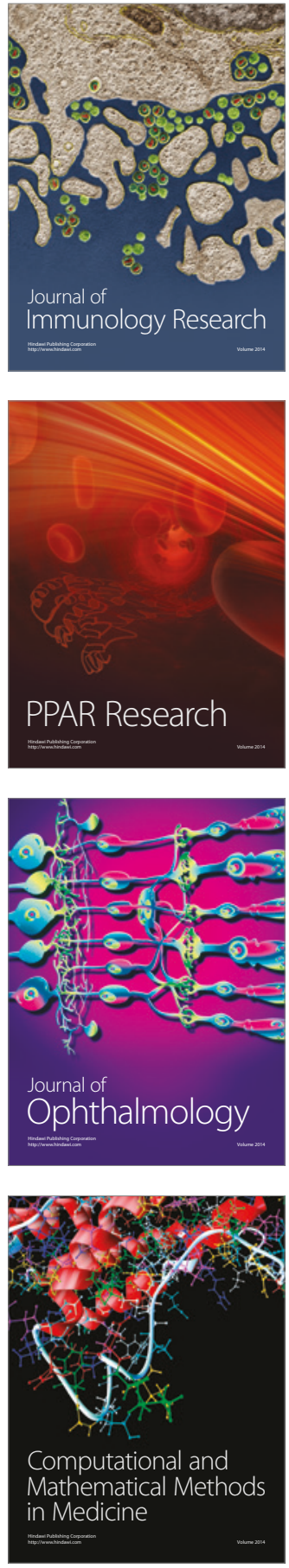

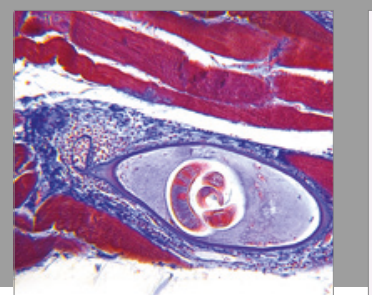

Gastroenterology Research and Practice
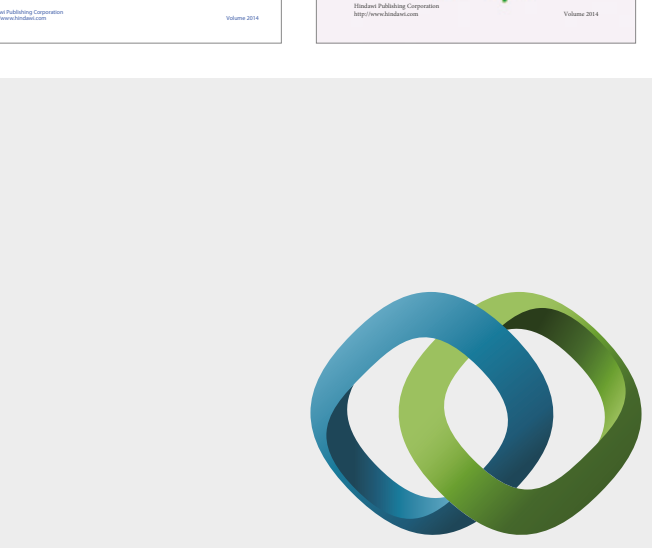

\section{Hindawi}

Submit your manuscripts at

https://www.hindawi.com
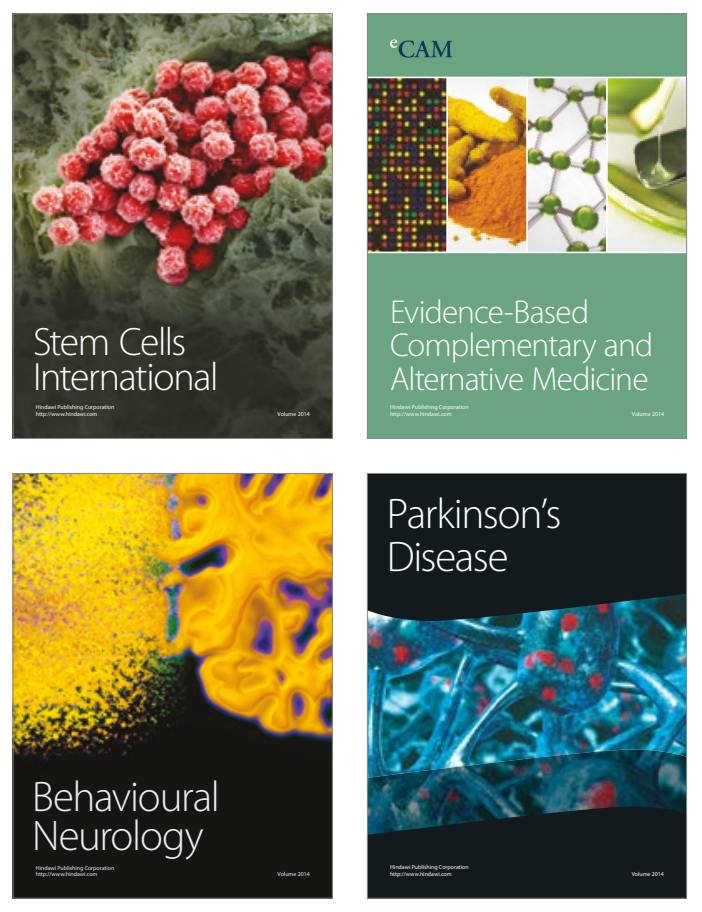
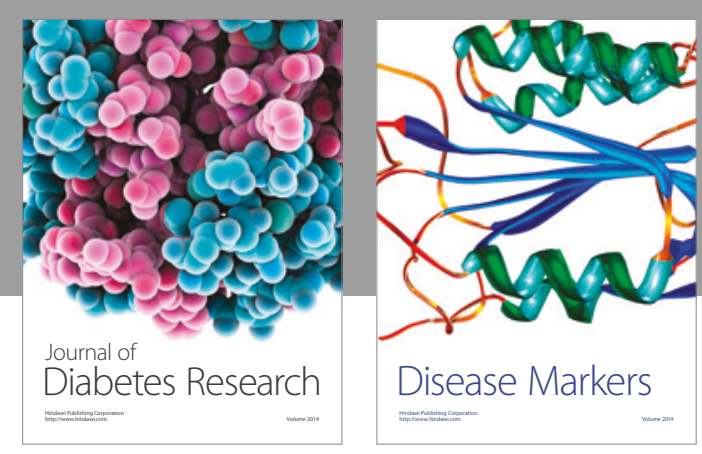

Disease Markers
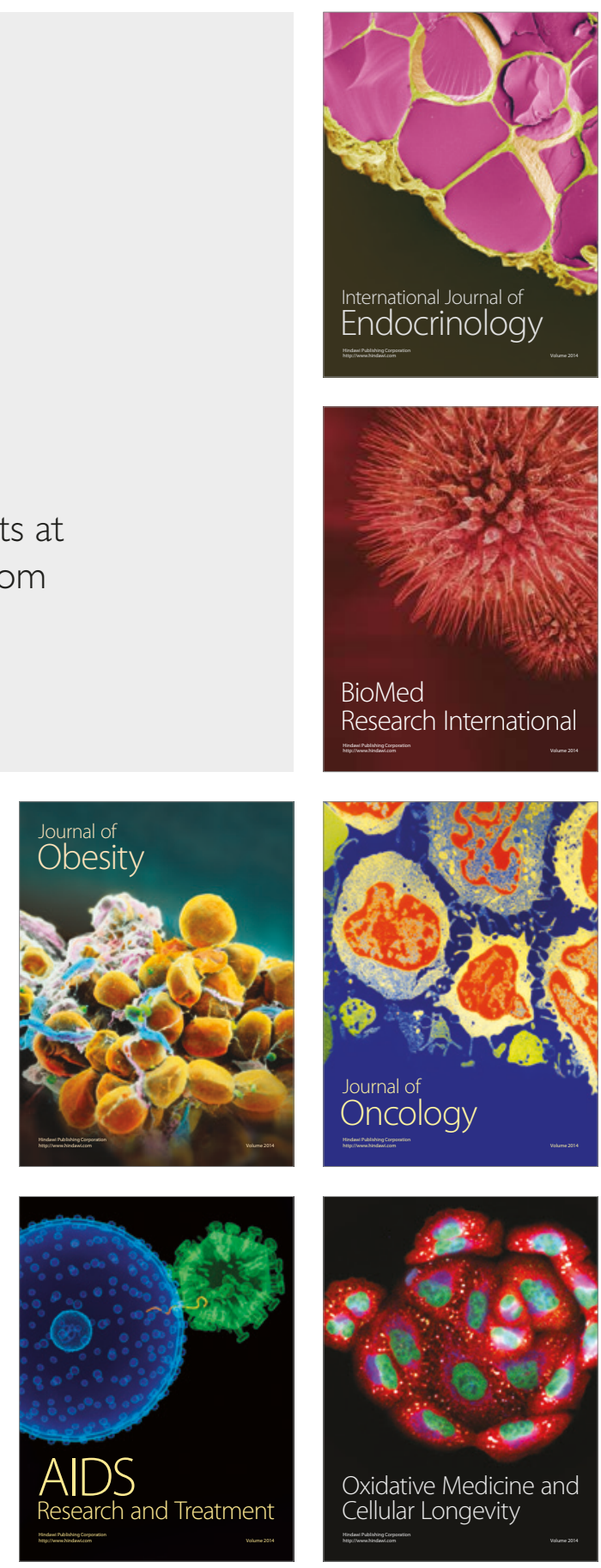